\title{
An Approach towards Balanced Energy Consumption in Hierarchical Cluster-based Wireless Sensor Networks
}

\author{
Plamen Z. Zahariev, Georgi V. Hristov and Ivanka D. Tsvetkova \\ Department of Telecommunications, University of Ruse "Angel Kanchev", Bulgaria
}

\begin{abstract}
In this paper we investigate the mechanisms for organization of the clusters in wireless sensor networks (WSN). After the short introduction to these systems we present the communication architecture and the energy dissipation model, which are used for the hierarchical cluster-based WSN. In the third section of the paper we analyze the current widely used process for organization of the clusters and we outline its main disadvantages. Later we present an approach for balanced consumption of the energy by the sensor motes, and then we propose a modification to this approach. In the next section we present and analyze the results of a series of simulation experiments, which we have conducted with the proposed approach, and then we compare these results with the ones obtained from the simulation experiments, which are conducted with the other presented approaches.
\end{abstract}

Keywords: wireless sensor networks, balanced energy consumption, hierarchical organization

\section{Introduction}

The modern wireless sensor networks combine the ability for sensing the environment, the possibility for local data processing and transmission of the information through the wireless medium. These networks are a powerful tool, characterized by their many capabilities, and are suitable for a large variety of applications, like agricultural and fishery purposes, observation of animal movement and migration, vehicle tracking, people locomotion and health monitoring and other. The process of designing wireless sensor networks requires knowledge of both their characteristics and the limitations of the underlying hardware components. Each of the devices in these networks has to provide a set of characteristics, which will affect the network that will emerge as they are deployed. At the same time, these devices have to meet the requirements for size, cost and power consumption that lay in the very definition of this type of networks.

A different set of challenges is to develop suitable network architecture and energy efficient data delivery protocols, which will correspond to the individual device capabilities, requirements and actions. The most widely adopted architecture for wireless sensor networks is the hierarchical cluster-based architecture. The most widely used protocols have mainly adopted this architecture and are developed with the idea for energy efficiency. These protocols are closely related in their nature and do not rely on a more detailed study of the approaches for organization of the clusters and their implementation in the wireless sensor networks.

We believe that a comprehensive study of the cluster organization processes for wireless sensor networks will support the development of new protocols, which will provide better energy efficiency and by that a longer and stable operational lifetime of the devices in the networks.

\section{Communication Architecture and Radio-energy Dissipation Model of the Hierarchical Cluster-based WSNs}

The communication processes in the wireless sensor networks are characterized by the huge consumption of energy [1]. Based on the hierarchical approach, for network organization every 
WSN can be presented by a two layered hierarchical model in which the communication processes at Layer 1 involve the sensor devices and the motes controlling the clusters - the cluster heads, while the second layer presents the communication processes between the cluster heads and the base station (Figure 1).

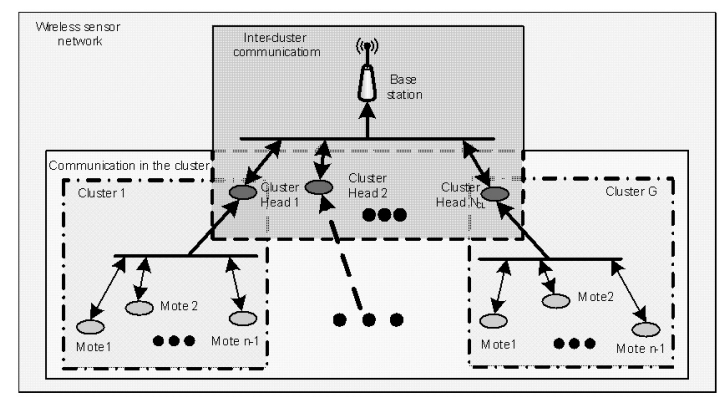

Figure 1. Two layered architecture of a hierarchical wireless sensor network with the intra-cluster communication processes at Layer 1 and the inter-cluster communication processes at Layer 2.

In order to present the amount of the energy, which is needed for communication purposes, we can use the radio-energy dissipation model from Figure 2. Based on this model, the transmitter will use a combined amount of energy consisting of the amount of energy needed by the radio electronics $-E_{\text {elect }}$ and the amount of energy needed by the radio amplifier $-E_{A}$. The receiver will need only energy for powering the electronic components of its radio interface.

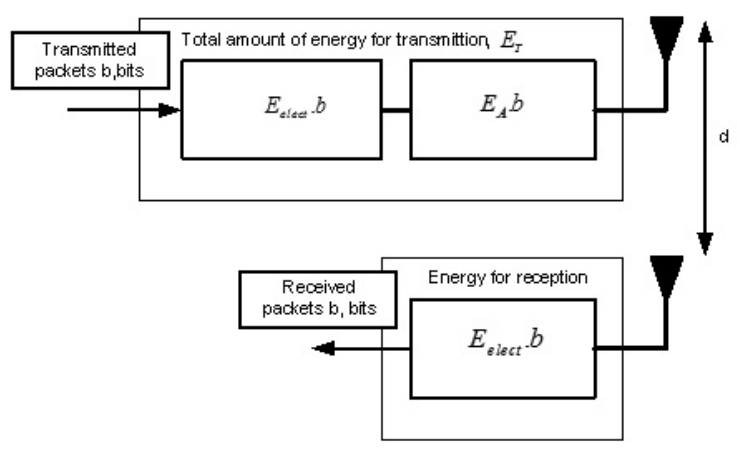

Figure 2. Radio-energy model used for describing the communication processes in the WSNs.

The amount of energy needed for the reception $\left(E_{R X}\right)$ and the transmission $\left(E_{T X}\right)$ of a packet with size of $b$ bits at the distance of $d$ meters is equal to:

$$
E_{R X}=b E_{\text {elect }},
$$

$$
E_{T X}=b\left(E_{\text {elect }}+E_{A} d^{k}\right)
$$

where $k$ is a coefficient used to present the signal strength loss [2]. The value of this coefficient depends on the data propagation model used in the system. For free space and direct line of sight $k=2$ and for environments with severe interference $k=4$.

If we assume that in the investigated WSNs the data delivery is performed by the means of direct communication and by using (1) and (2) we can calculate the amount of energy used for the communication processes by the sensor motes and by the cluster heads.

If every cluster in the network consists of $j$ sensor nodes and according to (2), the amount of energy used for the transmission of a packet with size of $b$ bits by every sensor mote in the cluster is equal to:

$$
E_{T X n_{i}}=b\left(E_{\text {elect }}+E_{A} d_{n_{i} C H}^{k}\right)
$$

where $n_{i}$ is a random sensor mote, with $i \epsilon[1, j]$, and $d_{n_{i} C H}$ is the distance between the cluster head $C H$ and $n_{i}$.

Similarly by applying (1), the amount of energy, needed by the cluster head $\mathrm{CH}$ for the reception of the packets, send to it by all sensor motes in the cluster, will be equal to:

$$
E_{R X_{C H}}=j b E_{\text {elect }} .
$$

The total amount of energy used for the communication processes in every cluster $E_{c c}$ is:

$$
E_{c c}=E_{n}+E_{C H}=\sum_{i=1}^{j} E_{n_{i}}+E_{C H},
$$

where $E_{n}$ and $E_{C H}$ are respectively the amount of energy needed for the communication processes by the sensor motes and by the cluster head. $E_{n}$ and $E_{C H}$ can be expressed by (3) and (4). This can be explained with the fact that the sensor motes send their packets directly to the cluster head and do not receive any packets and since we investigate the communication processes in the cluster only, then the cluster head is only receiving packets.

Since the base station is assumed to be energy independent, the energy needed for inter-cluster 
communication is equal to the sum of the energy needed by every cluster head. If we have a total of $G$ clusters in the network, then:

$$
E_{i c c}=\sum_{i=1}^{G} E_{C H_{i}}
$$

If we assume that every wireless sensor network can be presented as $N$ sensor nodes, divided equally in $G$ clusters, such that for every cluster the number of nodes is $n_{j}=N / G$, then the energy needed for communication in the network can be given by:

$$
E_{\text {comm }}=\sum_{i=1}^{G} E_{c c_{i}}+E_{i c c}
$$

where $E_{c c}$ and $E_{i c c}$ are respectively the amount of energy needed for intra-cluster and intercluster communication.

\section{Current State of the Cluster Organization Process and Problem Definition}

Currently one of the biggest problems in the WSNs is the fact that the sensor devices are battery powered. This means that the motes are in possession of a limited amount of energy, and, by that, their lifespan is also limited. In order to minimize the amount of energy, which is needed for communication processes, many protocols for WSNs are using a distance-based organization approach. As seen in Section 2 of this paper and by analyzing (2), we can clearly see that the amount of energy needed for the transmission of a packet is related to the size of the data and the distance the packets must travel $\left(E_{\text {elect }}\right.$ and $E_{A}$ are assumed constants and depend on the type of hardware used for the sensor devices). In this sense, minimizing the amount of energy, which is needed for transmission can be achieved by either decreasing the size of the transmitted data or by decreasing the distance it must travel. Some WSNs are information dependant and the data cannot be aggregated and/or compressed, so for these networks the only possible option remains the decrease of the distance between the source of the data and the sink. Currently, there are several widely known protocols which are being used in the hierarchical WSNs $[1,2]$, but they are all using the distance-based approach for cluster organization, which can be described by the following pseudo code:

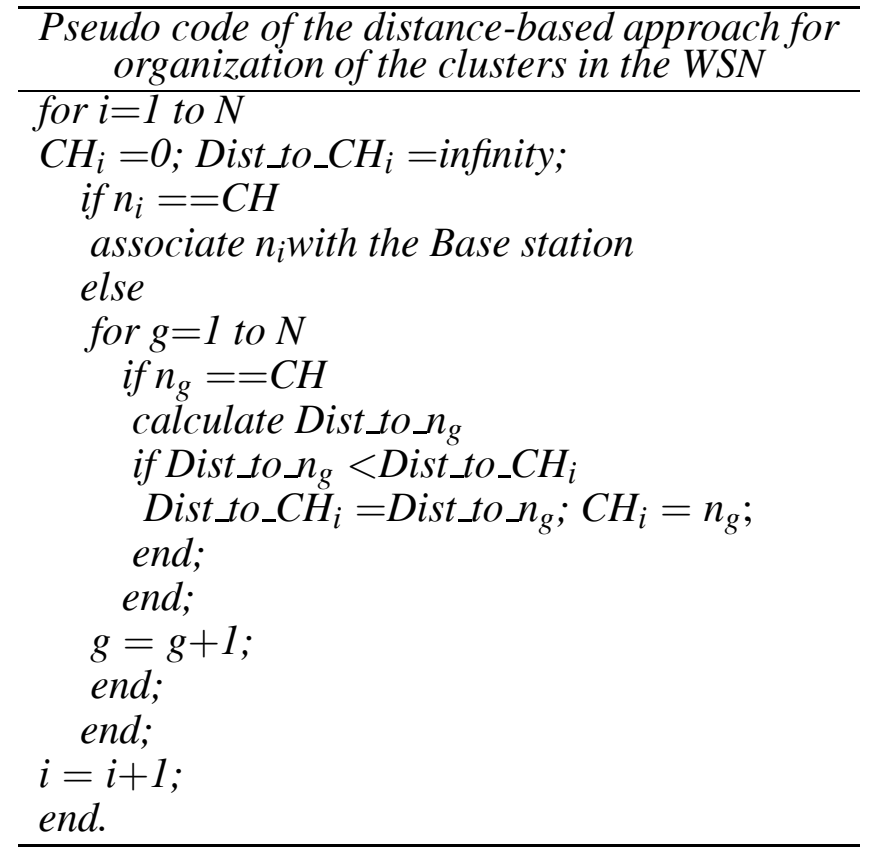

As seen by the pseudo code above, the sensor nodes will connect to the closest cluster head in their vicinity.

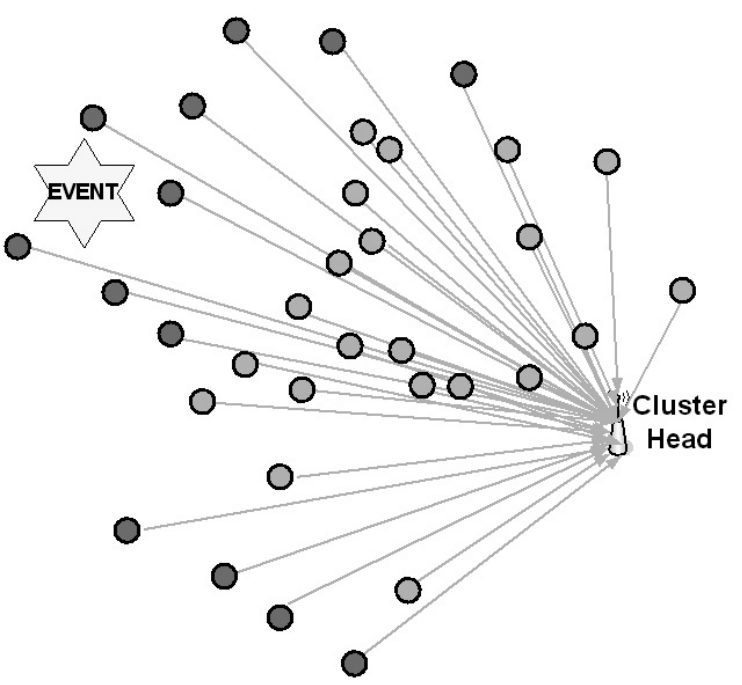

Figure 3. Unbalanced energy consumption as a consequence of the various distances between the motes and the $\mathrm{CH}$. The red sensor motes have exhausted their energy and are unable to detect the occurring event.

This approach will result in significant increase of the overall lifetime of the devices in the networks due to the more efficient consumption 
of the energy for the communication processes. This is the main reason for the wide usage of this approach. Nevertheless, distance-based cluster organization protocols will also lead to the unequal consumption of the energy by the devices in the network. This is a consequence of the variable distances between the nodes and the cluster heads, and can lead to some problems, especially in networks with low sensor redundancy or in networks, which have to provide high level of confidence of the measured readings (Figure 3).

\section{An Approach for Organization of the Clusters with Balanced Energy Consumption}

The amount of the energy and the balanced energy consumption are the two main factors, which have to be considered when designing communication protocols for wireless sensor network. The amount of energy is a parameter, which depends entirely on the characteristics of the batteries of the devices and corresponds to the device lifetime. The balanced energy consumption is a characteristic, which is connected to the amount of energy, necessary for the normal operation of the devices in the cluster for a given period of time and is responsible for the higher reliability of the network. Only one of these factors is not enough for the determination of the best possible cluster head. This is the major disadvantage of the distance-based approach for cluster organization in the WSNs.

If all sensor motes implement a complex parameter for evaluation of the cluster heads, and if this parameter is a function of the amount of energy needed for the communication processes and the available amount of energy, then this will lead to the election of a cluster head, such that a fairly balanced consumption of the energy can be achieved. By implementing this approach, the complex parameter for evaluation of the routes between every two sensor motes $n_{a}$ and $n_{b}$ can be defined as:

$$
W\left(n_{a}, n_{b}\right)=\frac{E_{c o m}\left(n_{a}, n_{b}\right)}{E_{n_{a}}}
$$

where $E_{\text {com }}$ is a function, which is defining the amount of energy needed for the transmission of the data between both nodes, and $E_{n_{a}}$ is the amount of energy in the battery of node $n_{a}$ at the beginning of the cluster organization process.

Pseudo code of the presented approach can be given as:

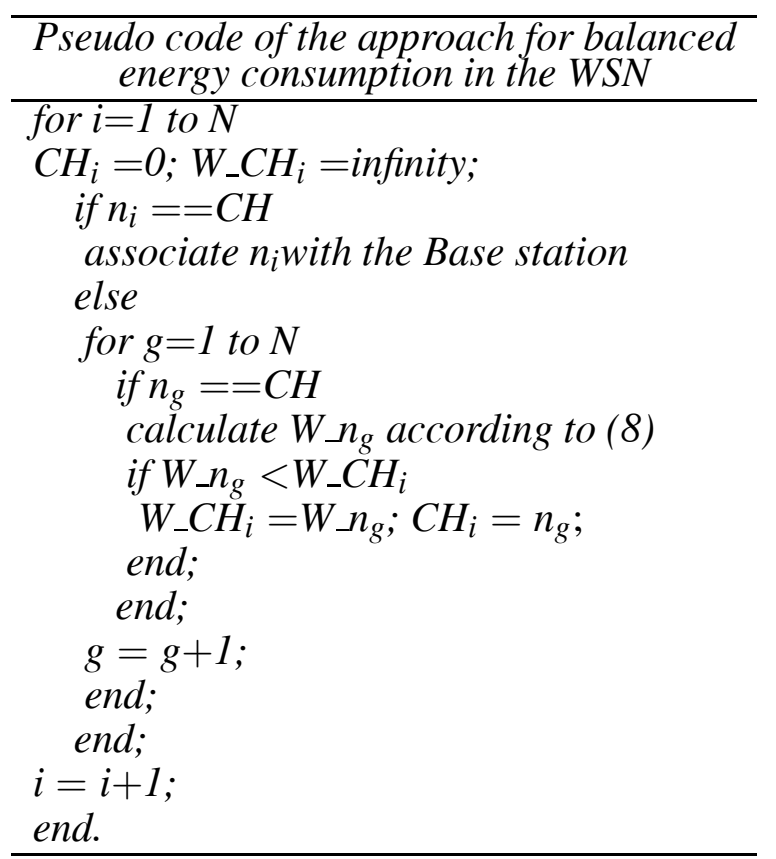

\section{Modification of the Approach for Organization of the Clusters with Balanced Energy Consumption}

The approach for organization of the clusters with balanced energy consumption can guarantee at a certain level that the lifetime of every device in the network will be the same. Nevertheless, due to the fact that the abovementioned approach does not consider the amount of the energy in the receiving device, the total lifetime of the network is severely decreased. The modification of the approach for organization of the clusters for the purpose of longer total lifetime, while at the same time having a fairly distributed consumption of the energy by all devices in the network, will lead to an overall increase of the performance of the wireless sensor network. This mechanism can be improved by using the amount of the energy in the sinks as well. This will guarantee a fairer distribution of the network traffic and, by that, a fairer distribution of the consumption of the energy as well.

Analyzing any WSN, then for every three sensor motes $n_{a}, n_{b}$ and $n_{c}$, such that $n_{a}$ is a sensor 
mote and $n_{b}$ and $n_{c}$ are cluster heads, we can say that $n_{a}$ will associate with $n_{b}$ if and only if $W\left(n_{a}, n_{b}\right)<W\left(n_{a}, n_{c}\right)$, where $W\left(n_{a}, n_{b}\right)$ can be defined as:

$$
W\left(n_{a}, n_{b}\right)=\frac{E_{c o m}\left(n_{a}, n_{b}\right)}{E_{n_{a}}} \frac{E_{R X}}{E_{n_{b}}}
$$

Introduction of the ratio between the amount of energy needed for the reception of the data by the sink and the available amount of energy in this device will guarantee the selection of the most appropriate cluster head, which will lead to the effective consumption of the energy for communication and, at the same time, the traffic will be fairly distributed among the motes. Despite the fact that the selected cluster head will possibly not guarantee the most effective energy consumption, it will guarantee the fair distribution of the traffic and, by that, the fair consumption of the energy by all devices in the network, thus providing a longer overall lifetime of the network devices. The pseudo code of the modified approach is the same like the pseudo code of the approach for balanced consumption of the energy, with the difference that the coefficient $W \_n_{g}$ is calculated using (9) instead of (8).

\section{Simulation Evaluation and Comparison of the Approaches for Organization of the Clusters in Hierarchical Cluster-based WSNs}

For the comparative analysis between the approaches for organization of the clusters we denote with M1 the standard mechanism from Section 3, with M2 the approach for organization of the clusters with balanced consumption of the energy from Section 4 and with M3 the modified approach for organization of the clusters with balanced consumption of the energy from Section 5. The simulation studies are performed using MatLAB models of the approaches which are applied to a wireless sensor network, placed on a field of $100 \times 100$ square meters. We have conducted two series of experiments with 25 and 100 sensor motes per cluster. For all simulations we assume that the base station is placed at $X=100 \mathrm{~m}, Y=100 \mathrm{~m}$ (on the edge of the cluster). The amount of the energy in the sensor motes, which is presented in the figures, is an average of 100 simulation runs and is measured during the 300-th communication round. For the simulations we assume size of the packets $b=512$ bits. In order to evaluate the mechanisms for organization of the clusters, we use the energy model from Section 2, where the energy needed for the function of the radio amplifier is $E_{A}=10 \mathrm{pJ} / \mathrm{bit} / \mathrm{m}^{2}$ when $k=2$ and $E_{A}=13 \cdot 10^{-4} \mathrm{pJ} / \mathrm{bit} / \mathrm{m}^{2}$ when $k=4$ and the amount of the energy needed for the radio electronics is $E_{\text {elect }}=50 \mathrm{~nJ} /$ bit.

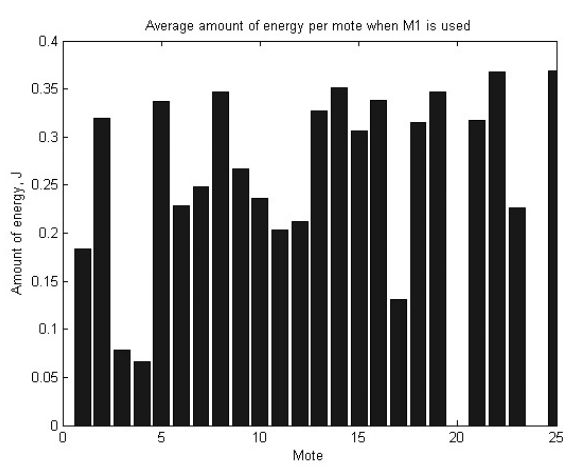

a)

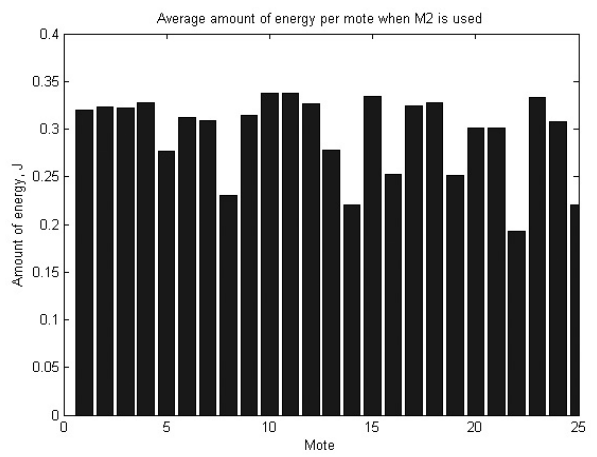

b)

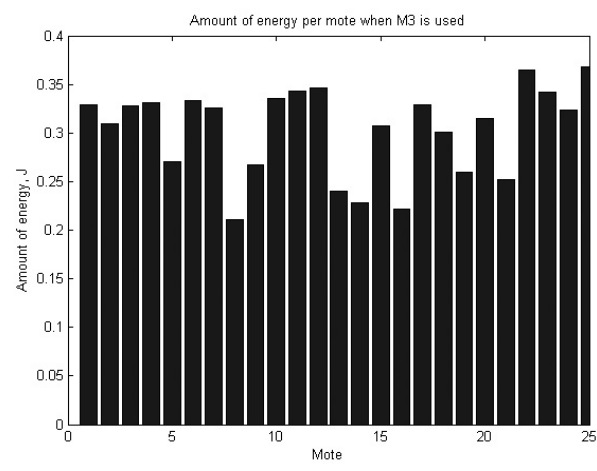

c)

Figure 4. Average amount of energy per sensor mote when M1 (a), M2 (b) and M3 (c) are used and there are 25 motes per cluster. 
As it can be seen in Figure 5, M1 is providing the longest operational time of the network, but some of the sensor motes start to deplete their energy much earlier than the systems where M2 and $\mathrm{M} 3$ are being used. On the other hand, the two approaches for balanced consumption of the energy provide stable operations of all nodes for a longer time, but at the cost of smaller overall lifetime of the cluster and the network respectively.

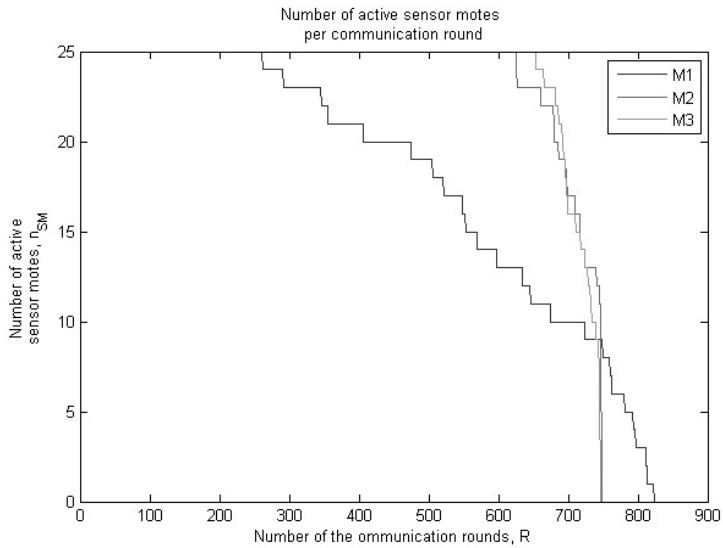

Figure 5. Average number of active sensor motes per cluster per communication round.

Comparing the results from Figure 4 and Figure 7 it is clearly seen that the overall amount of energy when M1 is used is larger than the amounts of energy when M2 and M 3 are being used, but the energy is not equally distributed among the sensors and this causes to some of the nodes to consume their energy much earlier than the rest. Comparing the results for the M2

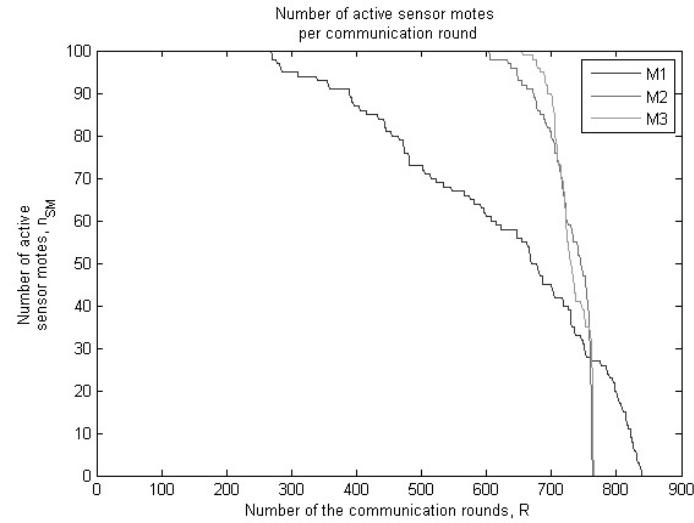

Figure 6. Average number of active sensor motes per cluster per communication round with 100 motes per cluster. and the M3 approaches, we can state that the modified approach (M3) performs slightly better. Proofs for this are the results from Figure 5 and Figure 6.

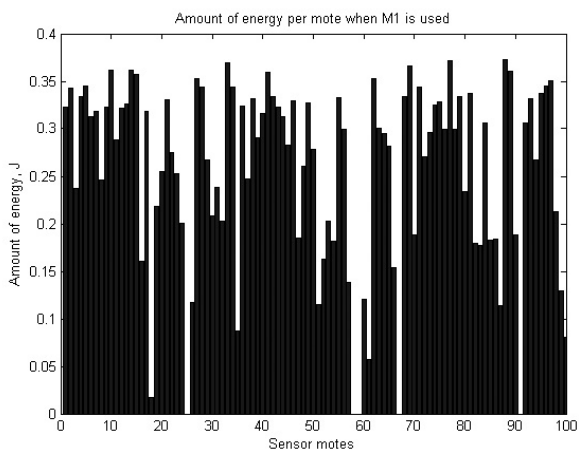

a)

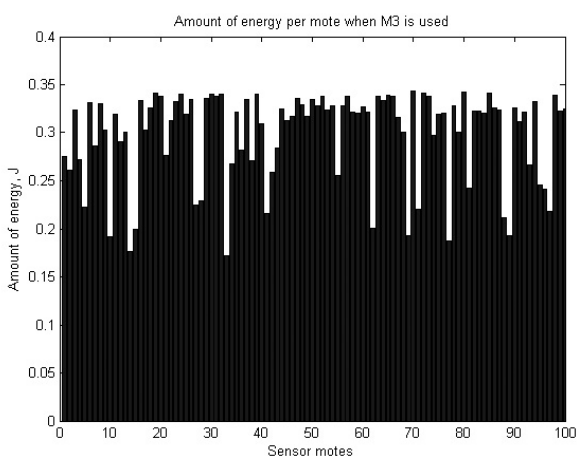

b)

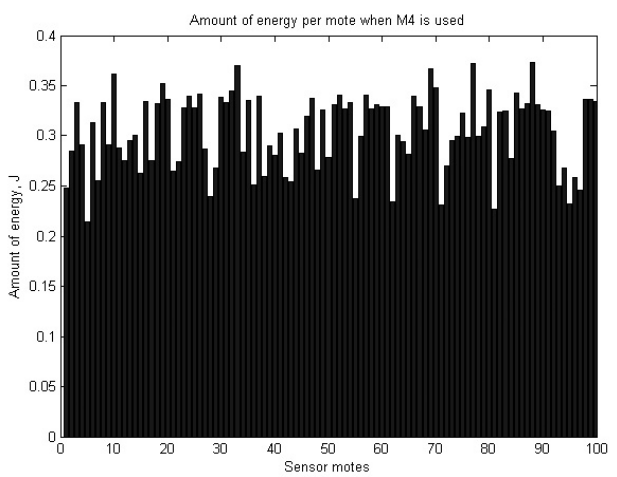

c)

Figure 7. Average amount of energy per sensor mote when M1 (a), M2 (b) and M3 (c) are used and there are 100 motes per cluster.

\section{Conclusions}

Based on the results from the conducted simulation experiments, we can state that the approaches for organization of the clusters with 
balanced consumption of the energy are most appropriate for implementation in the systems where a higher level of data reliability and accuracy are required. The M3 approach outperforms slightly M2 and provides longer average lifetime for the sensor devices, due to the fact that this approach takes into consideration the amount of the energy in the sink.

\section{Acknowledgments}

The work presented in this paper is completed as a partial fulfillment of project DMU-02/13-2009 "Design and performance study of an energyaware multipath routing algorithm for wireless sensor networks" of the Bulgarian Science Fund at the Ministry of Education and Science.
Received: June, 2012 Accepted: August, 2012

Contact addresses: Plamen Zahariev Departament of Telecommunications University of Ruse "Angel Kanchev" Studentska str. 8 Ruse Bulgaria e-mail: pzahariev@uni-ruse.bg

Georgi Hristov Departament of Telecommunications University of Ruse "Angel Kanchev" Studentska str. 8 Ruse Bulgaria e-mail: ghristov@uni-ruse.bg

Ivanka Tsvetkova Departament of Telecommunications University of Ruse "Angel Kanchev" Studentska str. 8 Ruse Bulgaria e-mail: itsvetkova@uni-ruse.bg

\section{References}

[1] G. Smaragdakis, I. Matta, A. Bestavros, SEP: A stable Election Protocol for heterogeneous wireless sensor networks. Proceedings of Int'l Workshop on SANPA, (2004).

[2] W. HEInZElman, A. ChandraKasan, H. BALAKRISHNAN, Energy-efficient communication protocol for wireless microsensor networks. Proceedings of the $33^{\text {rd }}$ Hawaii International Conference on System Sciences (HICSS-33), (2000).

PlAMEN ZAHARIEV was born in June 1983 in Ruse, Bulgaria. He received his BSc and MSc degrees in 2005 and 2007 from the Department of Computer Systems and Technologies at the University of Ruse. In 2011 he defended his $\mathrm{PhD}$ thesis in the field of wireless sensor networks and wireless communications and was appointed as assistant professor at the Department of Telecommunications at the University of Ruse. Dr. Zahariev has supervised more than $15 \mathrm{BSc}$ and MSc students. He has more than 8 years of experience in the field of telecommunication networks and has been a Cisco Academy Instructor for the last 5 years. $\mathrm{He}$ is a member of IEEE and has obtained the ACTi Associate level certificate. Dr. Zahariev is author and co-author of 1 book and more than 25 scientific papers presented at many international scientific conferences and published in several international journals. His main research interests are in the field of the wireless communication technologies, sensor and packet switched networks and telecommunication protocols.

GEORGI HRISTOV was born in February 1975 in Lovech, Bulgaria. He received his BSc and MSc degrees from the Department of Telecommunications at the University of Ruse, Bulgaria. Dr. Hristov obtained his $\mathrm{PhD}$ in the field of the communication networks and protocols in 2009. He was appointed as assistant professor at the Department of Telecommunications at the University of Ruse in 2008 and as associate professor at the same department in 2012. Dr. Hristov has supervised more than $25 \mathrm{BSc}$ and MSc students. He has more than 12 years of experience in the field of telecommunications and has obtained the CISCO CCNA, CAWFS, CCAI and the ACTi Associate level certificates. He is author and co-author of more than 50 publications and 4 books. His main research interests are in the field of the transmission and routing protocols, wireless communications, sensor networks and convergent systems.

IVANKA TSVETKOVA was born in June 1986 in the city of Lovech, Bulgaria. She received her BSc degree in 2008 and her MSc one in 2009, both in the field of the Telecommunication Systems from the University of Ruse "Angel Kanchev", Bulgaria. She is currently a PhD student in the Department of Telecommunications at the same University. Her research interests are in the field of wireless sensor networks, communication protocols and methods for positioning and localisation of mobile objects. 
